\title{
Visual Depth Perception of 3D CAD Models in Desktop and Immersive Virtual Environments
}

\author{
F. Gîrbacia, A. Beraru, D. Talabă, G. Mogan
}

\author{
Florin Gîrbacia, Andreea Beraru \\ Doru Talabă, Gheorghe Mogan \\ Transilvania University of Brasov \\ Romania, 500036 Brasov, Eroilor, 29 \\ E-mail: garbacia@unitbv.ro, aberaru@unitbv.ro \\ talaba@unitbv.ro,mogan@unitbv.ro
}

\begin{abstract}
:
In this paper is presented an experimental study that aims to compare the depth perception of virtual prototypes in immersive virtual environments with the depth perception of CAD models using 2D LCD display. First, a multipurpose solution of a large-scale interactive multi wall projected virtual environment named Holo-CAVE is described and then the conducted experiments are presented. The experiments carried out highlight that perceived depth values estimated for virtual prototypes are significantly influenced by the 3D stereoscopic visualization. Another interesting result of the study is that the estimated depth accuracy increases with the depth size that has to be perceived. The results of experimental study illustrate that the use of immersive stereoscopic visualization is useful during Computer Aided Design related activities.
\end{abstract}

Keywords: virtual reality, computer aided design, immersive 3D systems.

\section{Introduction}

Current Computer Aided Design (CAD) systems offer extremely rich modeling features and functions for the development of 3D virtual prototypes, which increase the productivity of the new products design. While the geometrical database is 3D since long ago, the user interaction within this software has not significantly changed. At present time CAD tools use standard WIMP (Window, Icon, Menu, Pointer) desktop-based Graphical User Interfaces (GUI), and the interaction is made through keyboard, mouse and CRT/LCD display which are solely 2D devices.

In the last years, Virtual Reality (VR) technology became a dynamic field of research that has began to be used to a certain extent in industrial applications. An important goal of the current worldwide research efforts is to facilitate the implementation of VR in industrial product development processes and to asses the impact and its feasibility into the workplace and everyday life contexts in terms of cost-effectiveness, human-machine interaction and side-effects on the users, as well as their impact on the actual working environment, at both individual and organizational level. VR provides new perspectives for user interaction with CAD tools. It enhances the immersion feeling and the depth perception of 3D objects, providing information with less perceptive ambiguities. This opportunity is important for a CAD application where users must have a direct and thus better appreciation of object shapes and dimensions. Many research activities are currently focused to integrate CAD architecture inside VR-systems in order to enhance the immersion feeling and the user interaction interface [1], [7], [10].

In many applications, VR technologies are used only for visualization and analysis of previously created CAD models [7], [10]. Another emerging category of VR design applications are the VR-CAD integrated systems, which allow the creation, modification and manipulation of 3D models directly in the VR environment [1]. Despite of the intensive research activities, none of them produced a significant impact for the development of the next generation of CAD systems. 
Therefore, it is necessary to develop various experimental researches in order to evaluate the impact of Virtual Reality technologies in the design process, and analyze their advantages and shortcomings. The last generation of commercial CAD systems still uses 2D CRT/LCD displays for visualization in most of the cases. The disadvantage of these devices is the lack of depth perception of 3D models.

Generally, the commercial visualization systems offer better visualization conditions comparing to the reduced possibilities of $2 \mathrm{D}$ displays. On the other hand, the performance of various technical solutions is different, each of them being appropriate to be used in special defined situations. Therefore, regarding CAD related activities, the development of an evaluation study of the visual perception is necessary, that will highlight the impact of VR technologies on visual perception of 3D CAD models during the design process of products.

There are previous extensive researches on depth perception of 3D models using immersive or volumetric 3D environments [3], [4], [5], [6], [8], [9], but the novelty of this experimental study is the comparison of the depth perception of CAD models using two distinct display modalities: monoscopic desktop system and 3D stereoscopic immersive environment.

The conducted experimental study analyzes the depth perception appreciation (value dimension along $\mathrm{Z}$ axis) of 3D CAD models using immersive CAVE-like stereoscopic visualization systems compared to the usage of $2 \mathrm{D}$ traditional display. It is known that the real perception of dimensions in Computer Aided Design related activities plays an important role in the decisionmaking process of a design solution. This experimental study is helpful in evaluating the benefits of immersive visualization system compared with monoscopic visualization using traditional $2 \mathrm{D}$ $\mathrm{LCD} / \mathrm{CRT}$ equipment.

\section{Description of Immersive Holo-Cave System}

The conventional CAD systems use for the visualization of the generated CAD model a traditional CRT/LCD 2D display. The disadvantage of this type of display for CAD systems is the lack of depth cues. Immersive Virtual Reality CAVE-like systems [2] are 3D stereoscopic displays that significantly improve the way users can visualize, navigate and interact in virtual environments. Compared to other devices like Head Mounted Display (HMD) or volumetric displays, the CAVE-like systems offer several advantages: improvement of the immersion awareness; obtaining high-definition stereoscopic images; large filed of view; collective visualization; collaboration between several users.

A multipurpose architecture was developed at VR lab of Transilvania University of Brasov, that is able to provide both possibilities for the 3D visualization: four walls CAVE-like and Holobench [12] functionality. Therefore, the system is called "Holo-CAVE" (figure 1). This solution allows making experiments related to the study of product engineering tasks that are performed by a human operator in the posture "seated" in the case of Holobench functionality or, alternatively, in a "standing" posture when the system is configured as CAVE. The developed system enables the visualization of large scale, high-resolution 3D stereoscopic images with a large field-of-view. Another advantage is the improvement of immersion awareness and the possibility to visualize the CAD models in their natural dimensions.

The physical structure of the Holo-CAVE has the dimensions of $2.8 \times 2.8 \times 3$ meters. The hardware architecture of the VR Holo-CAVE is presented in figure 2. The used screens were Screen-Tech type, rigid back-projection with the dimension of $2.7 \times 2$ meters. The mechanical frame of the system was constructed from wood material because it was the simplest and easiest construction free of magnetic field that could be built. The screens were attached to the frames using tubes and glue. Considering the high price and the variety of manufacturers, it was decided to use eight Hitachi CPX1350 high-end projectors (two for each screen for displaying the passive 


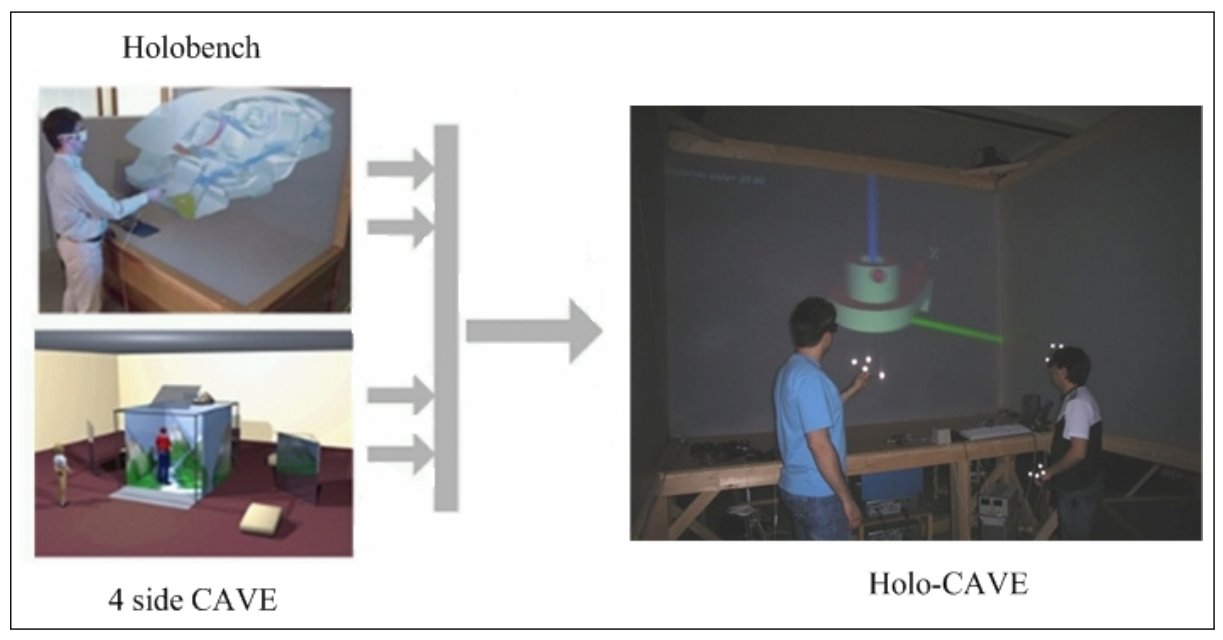

Figure 1: The Holo-CAVE immersive system

stereoscopic images) capable of displaying images with a resolution of $1400 \times 1050$. The physical projection distance between the projector and the screen is of 5.2 of meters. Mirrors have been used to cut down the required distance. In order to calculate the exact locations and dimensions of the mirrors and the projectors a CAD model was used. A PC Cluster was used as a computer system, composed of one server with a dual $2.4 \mathrm{GHz} \mathrm{CPU}$ and eight PC with $3 \mathrm{GHz} \mathrm{CPU}$ and dedicated video cards.

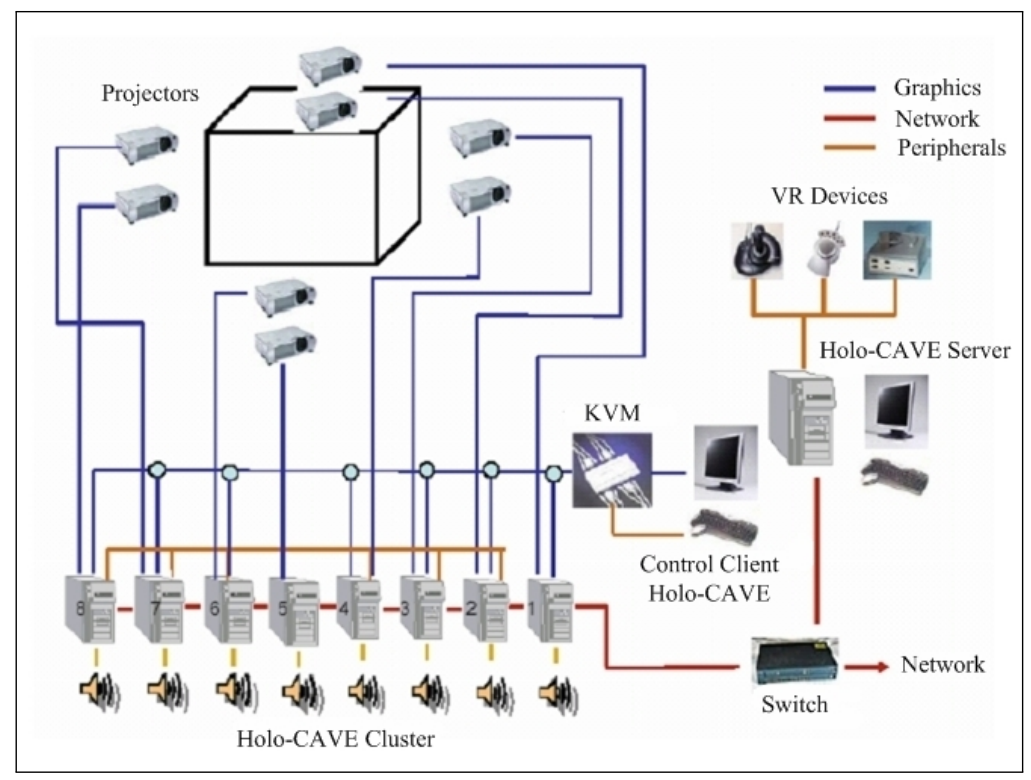

Figure 2: Architecture of the Holo-CAVE immersive system

The Holo-CAVE software is capable to load and display in a synchronized way a 3D scene on the multi-wall display environment, to display passive stereo 3D images and plug in different VR devices. It also provides methods by which the user can manipulate, add or remove objects in virtual environment. The Holo-CAVE software architecture is designed as a distributed highly modular network based on the strict separation of its VR system management into two layers: a Multi User Server that performs the administration of the 3D model, the users' interaction and a Virtual Environment Server that coordinates local projections and navigation devices. The 3D 
representation is full VRML2.0 (Virtual Reality Modeling Language [11]) thus compliant with all VRML sensors, events and sounds that can be used. Because VRML is the data format, VRML events are used for communication. Based on this approach, all VRML sensors, thus environmental sensors (time, proximity, visibility, and collision node), pointing device sensors (plane, cylinder, sphere, anchor, touch) and embedded JavaScript/ECMA Script can be used.

\section{Experiment Description}

This study tries to answer the following research questions:

1. Is immersive 3D visualization useful for the design engineers?

2. What is the performance of $2 \mathrm{D}$ display devices compared to 3D immersive visualization for perception of dimensions of 3D CAD models?

3. Which is the most intuitive and natural interface for the visualization of 3D CAD models?

We have devised and conducted two experiments to measure and record the estimated depth value of several CAD models using two types of displays. The former is the traditional desktop workspace with 2D input (keyboard and mouse) and 2D output (computer screen) peripherals. The latter consists of a multimodal immersive interface of an integrated VR-CAD system that uses the immersive 3D Holo-CAVE system. The results of these experiments will allow answering the three research questions.

\section{Experiment Procedure}

In order to evaluate the perception of depth in a CAD model an experiment was conducted, involving eight subjects (three women and five men) with the average age of 28 years and with a healthy sense of vision. None of the subjects used VR immersive stereoscopic 3D visualization for the perception of 3D CAD models before. Instead, they had extensive experience in using CAD software and good computer skills. In the conducted experiment six 3D CAD models were used, each of them composed from a parallelepiped part with variable dimensions. In order to give the subjects the opportunity of appreciating depth, the models were placed on a virtual table with the size of $300 \times 200 \times 150 \mathrm{~cm}$. The solid models were visualized using two types of devices: a universal 2D LCD display with the diagonal of 15.4" for desktop interface (figure 3a) and a Holo-CAVE system for immersive perception (figure 3b). To display the 3D environment in the first case a SolidWorks CAD system was used and in the second case dedicated software was used: BSContact Stereo VRML visualization player integrated in the Holo-CAVE. In the beginning, each subject was informed about the purpose of the experiment and specific instructions were given regarding the method of depth estimation. The subjects were asked to assess the depth of six objects using centimeter as measurement unit. In order to estimate the depth of CAD models, the subjects were informed about the size of the virtual table where virtual objects were positioned. In the case of Holo-CAVE immersive system, the distance between the viewpoint of the user and the projection screen was kept constant, $2 \mathrm{~m}$ (figure $3 \mathrm{~b}$ ). For each subject were displayed in a random order the CAD models. Each subject that participated to this experiment filled a questionnaire in which they were asked to provide information about age, experience of using VR equipment, experience in using CAD systems and computer skills.

Half of the users estimated first the depth of virtual objects using the traditional desktop CAD system, then, after a break of 20 minutes, they were asked to estimate the depth of virtual CAD objects using the Holo-CAVE stereoscopic visualization system. Simultaneously, the other half of subjects estimated first the depth of 3D CAD objects using stereoscopic system and then 
using monoscopic desktop system. The value of the estimated depth was recorded in a text file that was used afterward for the assessment of the results.

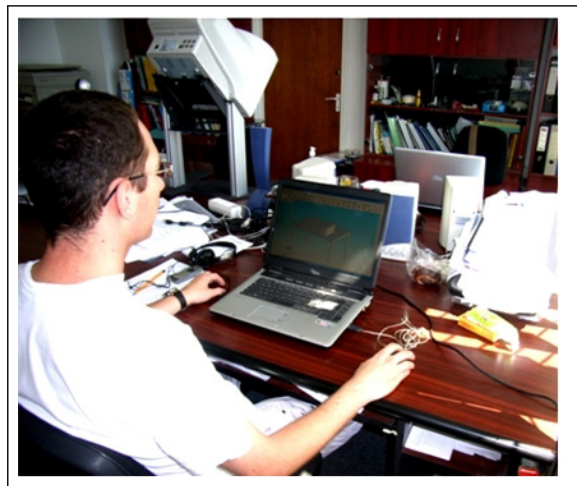

a)

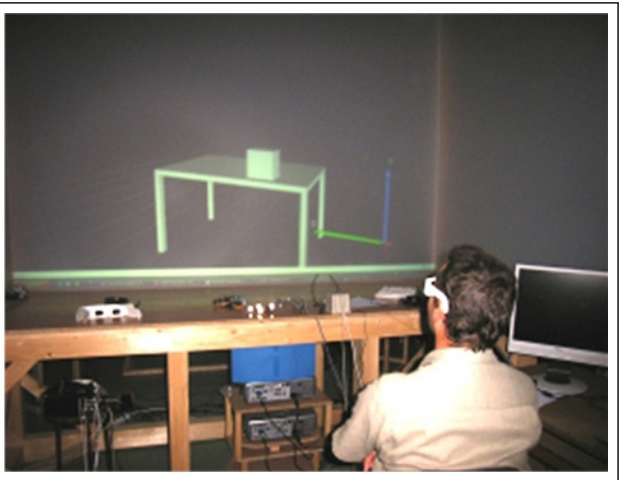

b)

Figure 3: The subject estimating depth using 2D display(a) The subject standing inside the CAVE-like visualization system(b)

\section{Results Evaluation}

Figure 4a presents the difference between the estimated values of the depth and the real dimensions of objects. After analyzing the data, the drawn conclusion is that for small values of depth (less than $35 \mathrm{~cm}$ ) the subjects overestimated the depth of CAD 3D models. Another significant result is that for higher values of depth, the average of estimated depth was more accurate when using stereoscopic 3D immersive visualization. An interesting result obtained by using the immersive 3D visualization, was that for all models the subjects overestimated the depth value.

Figure $4 \mathrm{~b}$ shows the accuracy of depth estimation that was obtained by using the value of relative error. The relative error was calculated using the following formula:

$$
E_{r}=\left(D_{p}-D_{r}\right) / D_{r}
$$

in which $\mathrm{Er}$ - is the value of the calculated relative error, Dp - the value of the estimated depth, Dr - the real value of virtual objects depth.

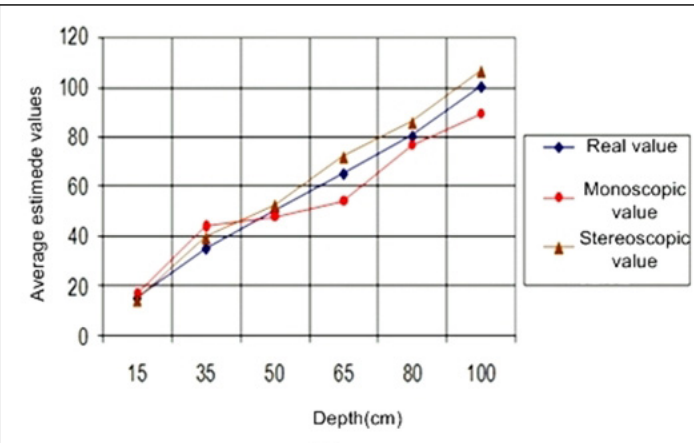

a)

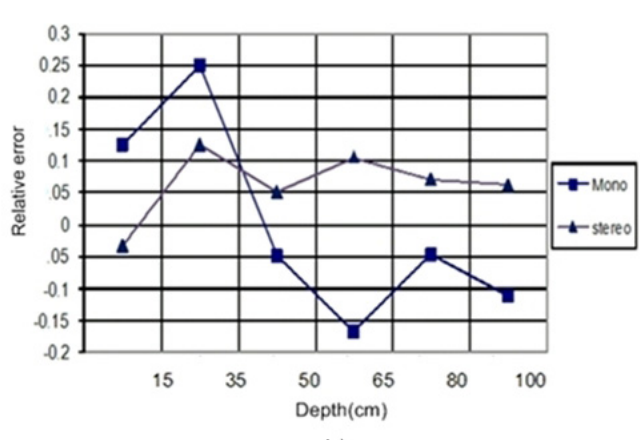

b)

Figure 4: Difference between the estimated values of the depth and real object dimensions(a); Accuracy of depth estimation(b) 
If the value of relative error is positive, the subject overestimated the depth of the CAD models, and if the value of relative error is negative then the subject underestimated the depth of the CAD models. The highest value of the relative error was obtained for the depth value of $35 \mathrm{~mm}$ and was due to the overestimation of depth. The conclusion drawn from this experiment is that the precision of depth estimation for stereoscopic viewing is lower for CAD models with small depth values, but increases significantly when CAD models depth value is higher.

After conducting the experiments, each of the subjects was asked what viewing equipment he/she prefers. Most subjects would use the immersive stereoscopic system because of the superior intuitive way of visual perception. However, few subjects considered as a shortcoming the need to wear glasses for passive stereoscopic visualization. We can conclude that the subjects estimated the depth of 3D CAD models with greater accuracy using the Holo-CAVE stereoscopic immersive visualization compared to monoscopic traditional desktop display. Another interesting result drawn from the experiment was the increasing of estimated depth with the dimensions of the 3D CAD models.

In order to emphasize the results of the experiments described above, there was a new series of experiments conducted on the same experimental set-up already presented, namely the Holo-CAVE system. The experiment was dedicated to assert the variation of stereopsis depth perception. The observers viewed the image by wearing polarizing glasses. The position of the observer was tracked by using a magnetic Ascension Flock of Birds tracking system with 6 DOF. The observer was standing inside the CAVE-like visualization system facing the screen (Fig. 5).

The viewing distance was set to three predefined values (1.5, 2.0, and 2.5 meters). The stereoscopic image consisted of two cubes, a red and a blue one having the sides of sizes 50 and $35 \mathrm{~cm}$ respectively. The arrangement of the cubes was such that the smaller one was set to a distance of $2 \mathrm{~m}$ (behind) with respect to the bigger one. The cube displayed to the left eye had a range of disparities added to it by shifting its horizontal position. The values of the disparities were 1,6 , and $11 \mathrm{~cm}$. When the observer fuses left and right image he always perceives the cubes being in front of the screen. Each observer was tested individually having the task to estimate the depth of the scene, namely which is the distance he perceives to the red cube and to the blue cube. The dimensions of the cubes and the relative position of one to the other were not made known to the participants. Free eyes movement and as much time as required to estimate the depth of the scene were allowed. Each observer for all values of the viewing distance and disparities repeated the task.

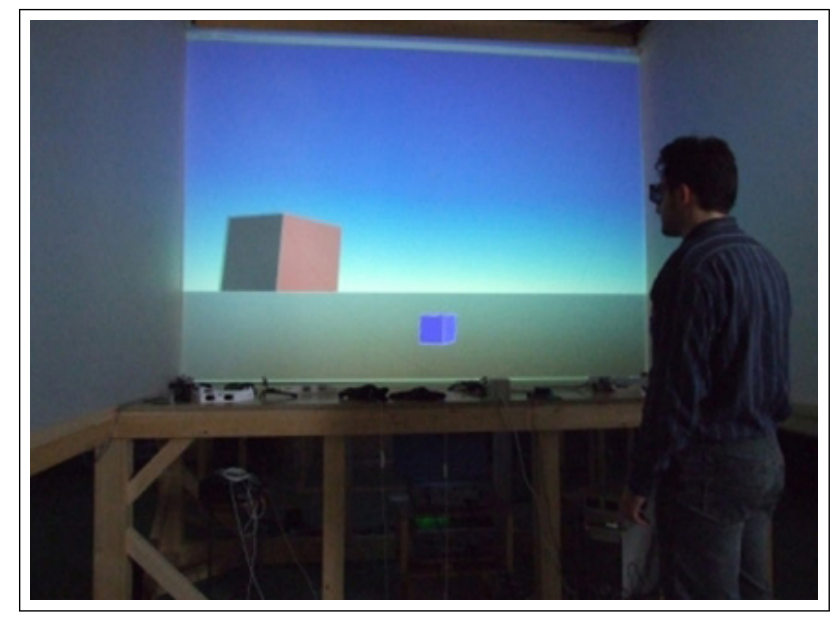

Figure 5: The observer standing inside the CAVE-like visualization system

Depth of a scene can be determined by using a simple arrangement as in figure 6 , where e 
is the interpupillary distance, $\mathrm{D}$ is the viewing distance, $\mathrm{d}$ is the disparity distance and $\mathrm{L}$ is the depth distance from the observer. The following equation expresses the depth $\mathrm{L}$ as function of variable d:

$$
L=e D /(e+d)
$$

For $\mathrm{D}$ and e constants, the depth is affected only by the variation of disparity d. Therefore, when disparity becomes smaller the object tends to be farther away from the user and vice versa. This is also illustrated in figure 7 where the predicted depth is represented as a function of the disparity distance for all the three cases of the viewing distance. For the calculation, an average value of the interpupillary distance of $6 \mathrm{~cm}$ has been considered.

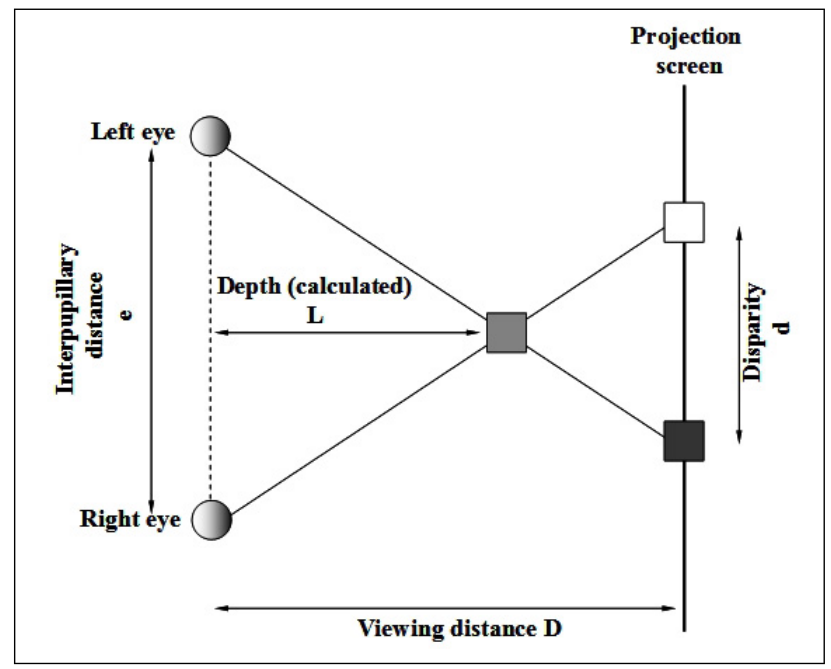

Figure 6: Determination of depth distance

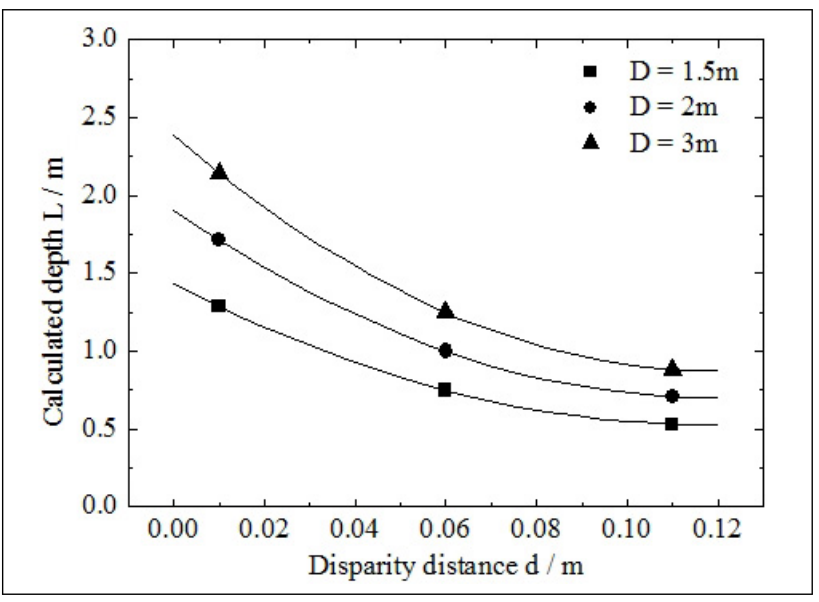

Figure 7: Calculated depth

The results of these experiments are summarized in figures 8a and 8b. The graph in figure 10 shows a good correspondence between the average values of the perceived depth as a function of disparity with the calculated values of the depth for the same value of the viewing distance $\mathrm{D}=2 \mathrm{~m}$. The open circles represent the average values of the perceived depth whereas the full circles are the calculated values of the depth. For $\mathrm{D}=1.5 \mathrm{~m}$, one can found the same good correspondence between the theoretically estimated values of depth and the perceived values. For 




Figure 8: Depth as a function of disparity(a);Depth as a function of the viewing distance(b)

$\mathrm{D}=2.5 \mathrm{~m}$ the agreement between theoretical and experimental values is not so good anymore, in this case the observers reporting difficulties in estimation of the depth.

In figure $8 \mathrm{~b}$ it is displayed the dependence of the perceived depth on the viewing distance for a constant value of the disparity $\mathrm{d}=1 \mathrm{~cm}$. For the sake of the comparison, the calculated depth is displayed too. The same trend it is observed for all the other values of the disparity. Concerning the precision of the depth perception of the viewer, it is observed that the users presented more accurate stereopsis when the value of the disparity is small while increasing the disparity value leads to more imprecise stereopsis.

\section{Conclusions and Future Works}

Realistic perception of the models depth in Computer Aided Design plays an important role in decision making of design engineers. In this paper was presented an experiment aimed to estimate the depth of 3D CAD models. From the performed experiment, we can emphasize that the perception of CAD model depth is significantly influenced by the stereoscopic visualization. The subjects estimated depth of 3D models with greater accuracy using the immersive stereoscopic Holo-Cave system compared to traditional desktop display. The accuracy of depth perception is not considerably improved when the depth of CAD models is small, but it increases significantly corresponding to a higher depth. As a general conclusion, we can declare that the alternative of replacing the $2 \mathrm{D}$ desktop systems with $3 \mathrm{D}$ VR visualization systems can be considered a viable alternative.

\section{Acknowledgments}

The research activities where supported by the Sectoral Operational Programme Human Resources Development (SOP HRD), financed from the European Social Fund and by the Romanian Government under the contract number POSDRU/89/1.5/S/59323.

\section{Bibliography}

[1] Bourdot, P.; Convard, T.; Picon, F.; Ammi, M.; Touraine, D.; Vezien, J.-M.(2010); VR-CAD integration: Multimodal immersive interaction and advanced haptic paradigms for implicit edition of CAD models, Comput. Aided Des, 42(5): 445-461. 
[2] Cruz-Neira, C.(1995); Virtual Reality Based on Multiple Projection Screens: The Cave and its Applications to Computational Science and Engineering, Ph.D. Dissertation, University of Illinois at Chicago, Chicago, IL, USA. UMI Order No. GAX95-32383.

[3] Foley, L. M.(1991); Stereoscopic distance perception, Pictorial communication in virtual and real environments, Stephen R. Ellis (Ed.). Taylor \& Francis, Inc., Bristol, PA, USA, 558-566.

[4] Grossman, T.; Balakrishnan R.(2006); An evaluation of depth perception on volumetric displays, Proceedings of the working conference on Advanced visual interfaces (AVI '06), ACM, New York, NY, USA, 193-200.

[5] Hoskinson, R.; Akai C.; Fisher, B.; Dill, J; Po B.(2004); Causes of depth perception errors in stereo displays, Proceedings of the 1st Symposium on Applied perception in graphics and visualization, ACM, New York, NY, USA, 164-164.

[6] Lang, M.; Hornung, A.; Wang, O.; Poulakos, S.; Smolic, A.; Gross, M.(2010); Nonlinear disparity mapping for stereoscopic 3D, ACM Transactions on Graphics, 29(4): 1-10.

[7] Raposo, A.; Soares, L; Wagner, G.; Corseuil, E.; Gattass, M.; Santos I.;(2009); Environ: integrating VR and CAD in engineering projects, IEEE Comput. Graph. Appl., 29(6): 91-95.

[8] Reichelt, S.; Haussler, R.; Futterer, G.; Leister, N.(2010); Depth cues in human visual perception and their realization in 3D displays, Three Dimensional Imaging, Visualization and Display, Bahram Javidi and Jung-Young Son(Ed.), Proc SPIE 7690, 134-144.

[9] Svarverud, E.; Gilson, S.J.; Glennerster, A. (2010); Cue combination for 3D location judgements, Journal of Vision, 10(1): 1-13.

[10] Weidlich, D.; Cser, L.; Polzin, T.; Cristiano, D.; Zickner, H. (2009); Virtual reality approaches for immersive design, Int. J. on Interactive Design and Manufacturing, 3: 103-108.

[11] www.web3d.org/x3d/specifications/vrml/ISO-IEC-14772-VRML97/

[12] http://www.barco.com/fr/virtualreality/product/961 